\title{
Clinical NIRS of the urinary bladder - A demonstration case report
}

\author{
A.J. Macnab ${ }^{\text {a,* }}$, R.E. Gagnon ${ }^{\mathrm{a}}$ and L. Stothers ${ }^{\mathrm{b}}$ \\ ${ }^{a}$ Department of Paediatrics, University of British Columbia, Vancouver, Canada \\ ${ }^{\mathrm{b}}$ Department of Surgery, University of British Columbia, Vancouver, Canada
}

\begin{abstract}
Urinary incontinence is a common affliction among people of all ages throughout the world. There are many causes of incontinence, treatment options are determined by the cause, and current diagnostic methods require urodynamic assessment, which involves urethral and rectal catheterization, which are uncomfortable and distasteful for patients. Since clinical near infrared spectrophotometry (NIRS) is a non-invasive, rapid means of measuring tissue oxygenation status at the bedside, we examined whether NIRS could be useful as a diagnostic tool for bladder dysfunction. An adult patient attending an incontinence clinic for routine urodynamic testing also had NIRS data collection during the standard bladder filling regimen. NIRS optodes were placed on the skin of the intact abdomen over the supra pubic region. Changes in oxy and de-oxy hemoglobin concentration and changes in cytochrome $\mathrm{c}$ oxidase net redox status via NIRS were collected at $6 \mathrm{~Hz}$. The magnitudes of change that occurred during NIRS data collection are on the order of $0.5 \mu \mathrm{mol} / 1$ and the moments of change correspond to the subject's reported sensations of bladder filling and emptying, and with conventional urodynamics. These observations suggest that NIRS may be a disruptive technology with a role to play in non-invasive evaluation of bladder dysfunction in humans.
\end{abstract}

\section{Introduction}

Modern clinical NIRS emerged in 1977 when Norris described the application of NIRS to the study of in situ human tissues, and Jobsis described the application of NIRS to the study of cerebral metabolism as related to the mitochondrial respiratory enzyme cytochrome c oxidase (Cyt), also known as cytochrome $\mathrm{a}_{1} \mathrm{a}_{3}[16,24]$. Today, there are more than a dozen commercial versions of clinical NIRS devices available for non-invasive transcutaneous sampling of oxygenated hemoglobin $\left(\mathrm{HbO}_{2}\right)$, de-oxygenated hemoglobin $(\mathrm{Hb})$ and Cyt redox status [4]. Clinical NIRS has been used to sample cohorts of all ages; and although intended as a cerebral monitor, it has been used to sample limb muscle, liver, breast, spine, tumours, lung, testis, spinal cord, kidney, heart, bowel and the splanchnic bed $[5,6,8-10,15,18-21,23$, $25,27,29,32,33]$.

Traditional urodynamic monitoring, the current technology for assessment of bladder dysfunction, measures the pressure exerted on the bladder by the accumulating urine during filling and measures the urine voiding rate and voided volume during emptying [34]. This technique requires invasive catheterization of both rectum and urethra [31]. Unfortunately, $40 \%$ of those with symptoms refuse testing because of embarrassment and fear of catheterization $[30,35]$. There is no NIRS counterpart for any of

\footnotetext{
${ }^{*}$ Corresponding author: Prof. Andrew Macnab, Director, Critical Care Research Office, Room L317, Mail Box 80, Children's \& Women's Health Centre, 4480 Oak St, Vancouver, BC, V6H 3V4. Tel.: +1 604875 3524; Fax: +1 604875 2890; E-mail: amacnab@cw.bc.ca.
} 
the traditional urodynamics urine measurements, and conversely there are no traditional urodynamics counterparts for any of the NIRS measurements [3].

As people age, their bladder capacity tends to decrease, leading to increased frequency of voiding (micturition), especially at night [17]. If the bladder neck and urethral sphincters are injured then they are not as effective at retaining the urine in the bladder (incontinence) [14]. The detrusor muscle itself may be unstable or overactive as a result of nerve damage or ischemia from either child-birth or previous incontinence surgery [12]. Anything that interferes with the parts of the brain involved in bladder monitoring can affect bladder function [28]. Anything pressing on the bladder can cause problems [26]. Cancer tumours can occur in the bladder wall and interfere with detrusor contraction [2]. Particulates in the urine can crystallize into stones within the bladder that then obstruct the urethra to hinder micturition [13,22]. In males, the prostate surrounds the urethra beneath the bladder, and if swollen can cause a constriction that hinders micturition [11]. In 1998, the World Health Organization (WHO) conservatively estimated that world-wide direct and indirect costs of urinary incontinence exceed US\$16 billion annually within the major pharmaceutical markets (USA, Top 5 European nations, and Japan). At that time, WHO estimated that bladder control problems affected more than 200 million people world wide.

In the urological setting, NIRS primarily interrogates the bladder wall, which consists of three layers: an outer layer (serosal) of connective tissue, a muscularis layer of smooth muscle (detrusor), and an inner mucous membrane comprised of transitional epithelial cells (urothelium) supported by a boundary of fibroblasts and sparse smooth muscle cells (lamina propria) [1,7].

This is the first report of successful interrogation of the bladder using NIRS non-invasively in a urology clinic.

\section{Method}

An adult human suffering from urinary incontinence was enrolled in a study approved by the university's Human Ethics Review Committee. The subject underwent urethral catheterization to facilitate routine urodynamic evaluation, including emptying and filling of the bladder via the catheter.

In this trial, NIRS was used in reflectance mode with the NIRS emitter and detector of a Hamamatsu NIRO-300 (Hamamatsu Photonics KK, Hamamatsu City, Japan), placed bilaterally on the intact skin surface above the midline of the in situ bladder. Data was collected at $6 \mathrm{~Hz}$ with $40 \mathrm{~mm}$ inter-optode spacing.

Following instillation of the catheter, the subject voluntarily voided and any residual urine volume was removed from the bladder by syringe withdrawal. NIRS was then initialized and data collection commenced. After a two minute stable resting baseline, bladder filling commenced via infusion pump at a rate of $1 \mathrm{ml} / \mathrm{min}$. The subject reported the first sensation of filling, the sense of urgency to void, and the sensation of being filled to capacity. The subject was then asked to attempt to prevent the bladder from leaking urine and shortly thereafter data collection was terminated to allow the subject to void.

\section{Results}

A typical NIRS adult human abdominal bladder $\mathrm{Hb}$ and $\mathrm{HbO}_{2}$ data collection is given in Fig. 1 .

A typical NIRS adult human abdominal bladder Cyt data collection is given in Fig. 2. Our Fig. 2, is a companion to Fig. 1; its cytochrome data was collected simultaneously with the hemoglobin data using the same NIRS device. 


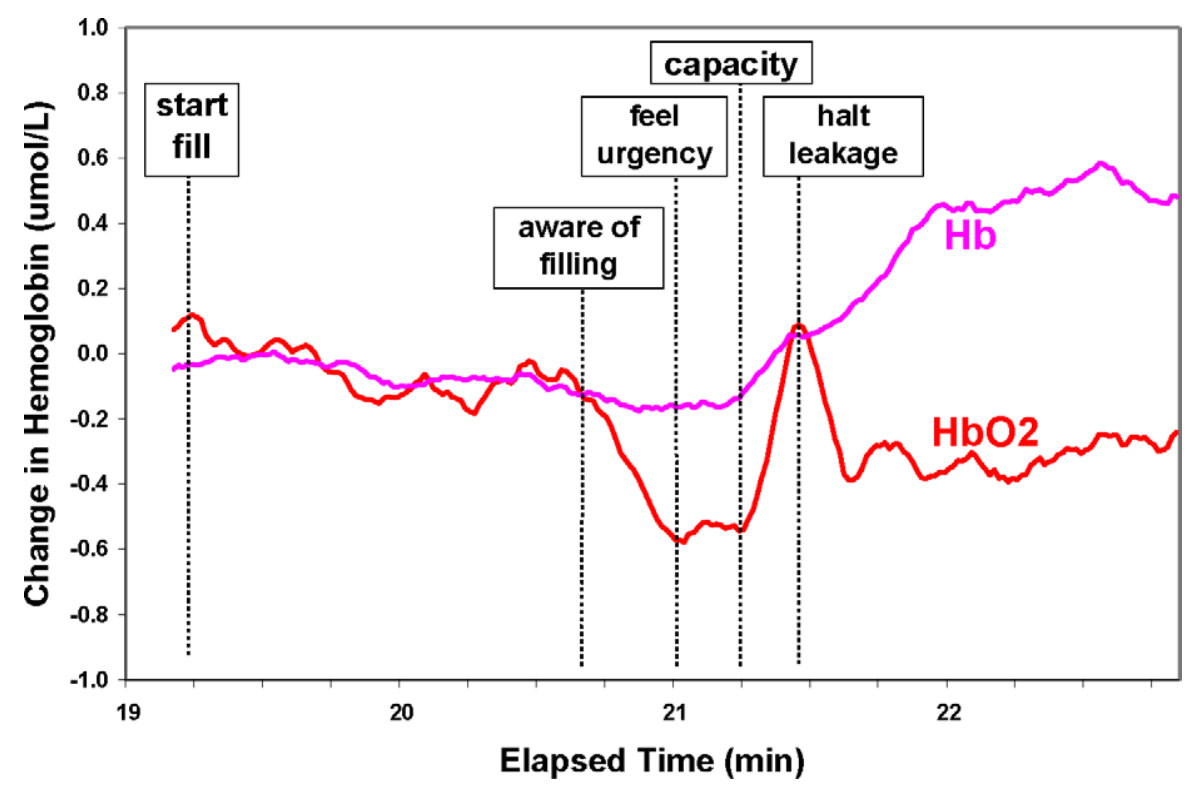

Fig. 1. Typical hemoglobin changes during controlled filling and emptying of the urinary bladder in a clinical urodynamics examination.

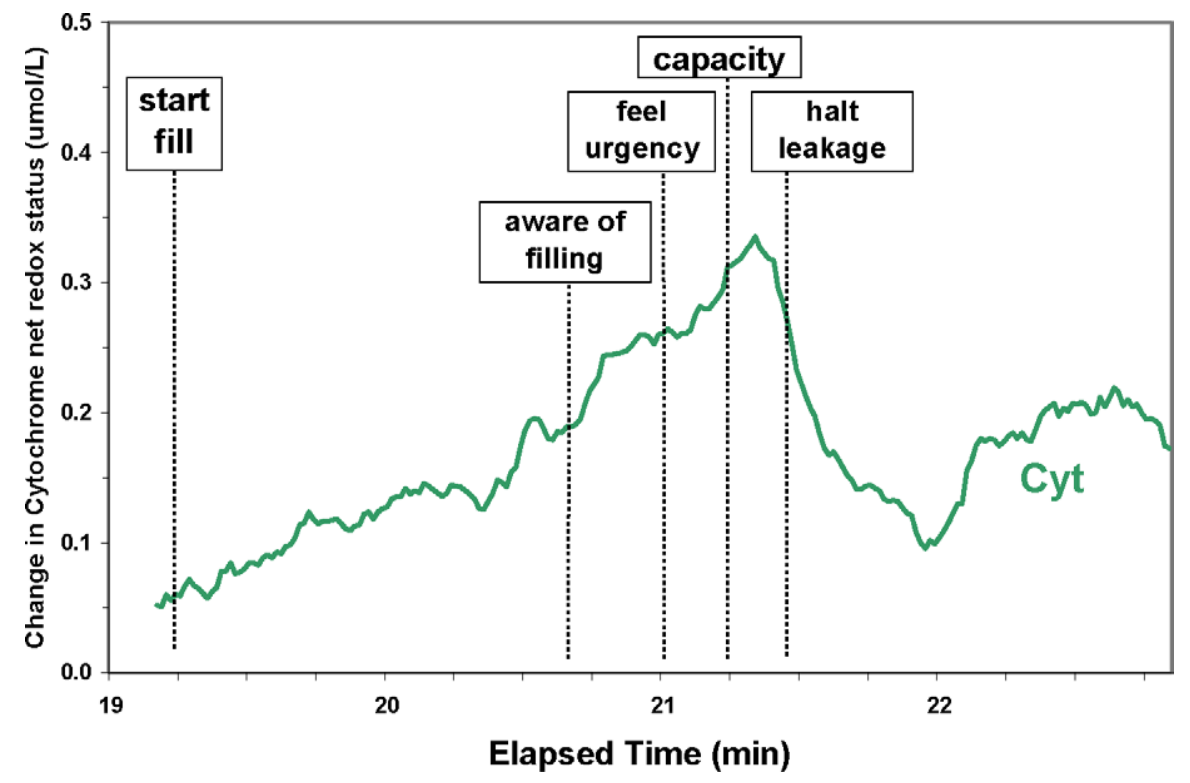

Fig. 2. Typical net oxidized minus reduced cytochrome c oxidase redox status changes during the same controlled filling and emptying of the urinary bladder as shown in Fig. 1.

\section{Discussion}

Figure 1 shows an adult human detrusor data collection with filling controlled by saline infusion pump. Interpretation of Fig. 1 indicates an immediate loss of blood volume in the bladder wall that is evident with the start of bladder filling, and that progresses until capacity is reached. The notations 
on the graph ("aware of filling", "feeling urgency", and "capacity") are the patient's comments. These markers suggest that NIRS might be able to serve as a biofeedback monitor. The graph notation "halt leakage" is when the subject began voluntary bladder sphincter contraction to attempt to retain urine. As this contraction continues, equal and opposite changes in concentration between oxy and de-oxy haemoglobin occur, probably as a result of the energy expenditure by the muscle consuming the oxygen. Although leakage is slowed initially, after 30 seconds the rate of leakage rises and there is an increase in detrusor blood volume as bladder volume decreases and wall thickness is restored.

Figure 2 illustrates concentration changes in the net oxidized minus reduced redox states of the intracellular respiratory chain enzyme, cytochrome c oxidase or cytochrome $\mathrm{a}_{3} \mathrm{a}_{3}(\mathrm{Cyt})$. Interpretation of Fig. 2 suggests that the diminished availability of oxygen (see $\mathrm{HbO}_{2}$ in Fig. 1) secondary to filling leads to an increase in the number of Cyt molecules retaining electrons. At the same stage, proton pumping in conjunction with the electron transfer is likely diminished thereby hindering adenosine triphosphate (ATP) synthesis, and by corollary, depleting energy stores. Oxygen availability and electron transfer are restored when leakage begins to occur after retention capacity is reached and vestibular pressure is reduced thereby permitting recovery of oxygenated blood volume in the resting bladder wall.

Importantly, there are portions of this human data collection (Figs 1 and 2) where NIRS data provides physiologic insight that the traditional urodynamics does not. One example that is noticeable is the 'halt leakage' graph point in Fig. 2 where there is a sharp drop in cyt absorbance detected. This observation could be due to the intensity of muscle work required in the voluntary attempt to halt leakage. Also, there are events detected via change in the cytochrome signal that are not evident in the haemoglobin pattern and vice versa. Hemoglobin resides in the blood, cytochrome resides in mitochondria. Hemoglobin provides a partial glimpse into cellular physiology by indicating whether oxygen has been delivered or depleted. In contrast, changes in the oxidation state of cytochrome c oxidase reflect more intricate physiology, indicating whether there was oxygen uptake, whether cells had switched from oxidative phosphorylation to substrate level phosphorylation, whether glucose stores are depleted, and whether ATP synthesis has become impaired. For this reason, a direct correspondence between changes in hemoglobin and changes in cytochrome is not expected.

Changes in cytochrome differ in quality from those in hemoglobin. Whereas hemoglobin reacts rapidly to changes in its environment so that a change can be quickly offset by some manipulation by a clinician, cytochrome balance is slower to respond, and thus is susceptible to over-correction. As a result, it is possible that if a change in the NIRS haemoglobin signal indicates an event that is having an adverse physiological effect and a corrective change is made that restores normality to the haemoglobin signal, the cytochrome pattern of change may still indicate that, in spite of improvement in the oxygen delivery (haemoglobin), cellular function is still being affected at the mitochondrial level.

In the single subject monitoring reported here, the increased reduction of cytochrome is likely due to voluntary muscle contraction depleting the ATP stores required to maintain effective muscle contraction. As a result of this demand for more ATP, higher rates of proton pumping within the cytochrome complex are required in synchronization with oxygen transit through the cytochrome complex. Thus, while cytochrome responds adequately to this ATP demand initially, cytochrome becomes reduced as $\mathrm{HbO}_{2}$ becomes depleted when the bladder volume increases and blood volume in the bladder wall decreases. Thus, the oxygen available as filling commences is progressively depleted and cytochrome becomes progressively reduced because there are fewer oxygen molecules to which to transfer its electrons. This is because the $\mathrm{HbO}_{2}$ available as the bladder filling commences becomes depleted as the bladder filling pressure rises, the volume of blood present in the bladder muscle decreases as the wall thing. Without 
restoration of the $\mathrm{HbO}_{2}$ concentration, a reduction in ATP production occurs with the result physiologically that the subject is no longer able to maintain the voluntary contraction and involuntary urination then follows.

\section{Conclusion}

Non-invasive NIRS monitoring can provide data from which physiologic aspects of bladder function, including mitochondrial metabolism in the detrusor muscle, can be inferred. This will provide advances in our ability to evaluate bladder function.

\section{Acknowledgement}

We wish to thank the St Paul's Hospital Foundation and its generous contributors in British Columbia for their support of our research.

\section{References}

[1] A. Aydin, R. Ucak, M. Karakok, M.E. Guldur and N.E. Kocer, Vascular plexus is a differentation criterion for muscularis mucosa from muscularis propria in small biopsies and transurethral resection materials of urinary bladder?, Int. Urol. Nephrol. 34(3) (2002), 315-319.

[2] P. Benedetti-Panici, M.A. Zullo, F. Plotti, N. Manci, L. Muzii and R. Angioli, Long-term bladder function in patients with locally advanced cervical carcinoma treated with neoadjuvant chemotherapy and type 3-4 radical hysterectomy, Cancer 100(10) (2004), 2110-2117.

[3] C. Blake and P. Abrams, Noninvasive techniques for the measurement of isovolumetric bladder pressure, J. Urol. 171(1) (2004), 12-19.

[4] B. Chance, C.E. Cooper, D.T. Delpy and E.O.R. Reynolds, Near-infrared spectroscopy and imaging of living systems, Philos. Trans. R. Soc. Lond. B Biol. Sci. 352(1354) (1997), 643-761.

[5] S.M. Cohn, J.E. Varela, G. Giannotti, M.O. Dolich, M. Brown, A. Feinstein, M.G. McKenney and P. Spalding, Splanchnic perfusion evaluation during hemorrhage and resuscitation with gastric near-infrared spectroscopy, J. Trauma 50(4) (2001), 629-634.

[6] W.N. Colier, F.M. Froeling, J.D. de Vries and B. Oeseburg, Measurement of the blood supply to the abdominal testis by means of near infrared spectroscopy, Eur. Urol. 27(2) (1995), 160-166.

[7] T. Congiu, R. Radice, M. Raspanti and M. Reguzzoni, The 3D structure of the human urinary bladder mucosa: a scanning electron microscopy study, J. Submicrosc. Cytol. Pathol. 36(1) (2004), 45-53.

[8] R.A. De Blasi, E. Quaglia, A. Gasparetto and M. Ferrari, Muscle oxygenation by fast near infrared spectrophotometry (NIRS) in ischemic forearm, Adv. Exp. Med. Biol. 316 (1992), 163-172.

[9] B. de Groot, C.J. Zuurbier and J.H. van Beek, Dynamics of tissue oxygenation in isolated rabbit heart as measured with near-infrared spectroscopy, Am. J. Physiol. 276(5 Pt 2) (1999), H1616-1624.

[10] A.D. Edwards, J.S. Wyatt, C. Richardson, D.T. Delpy, M. Cope and E.O. Reynolds, Cotside measurement of cerebral blood flow in ill newborn infants by near infrared spectroscopy, Lancet 2(8614) (1988), 770-771.

[11] R.M. Ellerkmann and A. McBride, Management of obstructive voiding dysfunction, Drugs Today (Barc.) 39(7) (2003), 513-540.

[12] C.H. Fry, Y. Ikeda, R. Harvey, C. Wu and G.P. Sui, Control of bladder function by peripheral nerves: avenues for novel drug targets, Urology 63(3 Suppl. 1) (2004), 24-31.

[13] F. Grases, O. Sohnel and A. Costa-Bauza, Renal stone formation and development, Int. Urol. Nephrol. 31(5) (1999), 591-600.

[14] M. Harrahill, Bladder trauma: a review, J. Emerg. Nurs. 30(3) (2004), 287-288.

[15] M. Hayashida, N. Kin, T. Tomioka, R. Orii, H. Sekiyama, H. Usui, M. Chinzei and K. Hanaoka, Cerebral ischaemia during cardiac surgery in children detected by combined monitoring of BIS and near-infrared spectroscopy, Br. J. Anaest. 92(5) (2004), 662-669. 
[16] F.F. Jobsis, Noninvasive infrared monitoring of cerebral and myocardial oxygen sufficiency and circulatory parameters, Science 198 (1977), 1264-1267.

[17] R. Kevorkian, Physiology of incontinence, Clin. Geriatr. Med. 20(3) (2004), 409-425, v.

[18] M. Kragh, B. Quistorff, E.L. Lund and P.E. Kristjansen, Quantitative estimates of vascularity in solid tumors by noninvasive near-infrared spectroscopy, Neoplasia 3(4) (2001), 324-330.

[19] A.J. Macnab, R.E. Gagnon and F.A. Gagnon, Near infrared spectroscopy for intraoperative monitoring of the spinal cord, Spine 27(1) (2002), 17-20.

[20] S.M. McGill, R.L. Hughson and K. Parks, Lumbar erector spinae oxygenation during prolonged contractions: implications for prolonged work, Ergonomics 43(4) (2000), 486-493.

[21] D.J. Mehagnoul-Schipper, B.F. van der Kallen, W.N. Colier, M.C. van der Sluijs, L.J. van Erning, H.O. Thijssen, B. Oeseburg, W.H. Hoefnagels and R.W. Jansen, Simultaneous measurements of cerebral oxygenation changes during brain activation by near-infrared spectroscopy and functional magnetic resonance imaging in healthy young and elderly subjects, Hum. Brain Mapp 16(1) (2002), 14-23.

[22] F. Millan-Rodriguez, C. Errando-Smet, F. Rousaud-Baron, F. Izquierdo-Latorre, A. Rousaud-Baron and H. VillavicencioMavrich, Urodynamic findings before and after noninvasive management of bladder calculi, BJU Int. 93(9) (2004), 12671270.

[23] T. Noriyuki, H. Ohdan, S. Yoshioka, Y.T. Asahara and K. Dohi, Near-infrared spectroscopic method for assessing the tissue oxygenation state of living lung, Am. J. Respir. Crit. Care Med. 156(5) (1997), 1656-1661.

[24] K.H. Norris, Light transmitted through human tissues, in: The Science of Photobiology, K.C. Smith, ed., Plenum Press, New York, 1977 , pp. 400-409.

[25] H. Ohdan, K. Mizunuma, H. Tashiro, D. Tokita, H. Hara, T. Onoe, K. Ishiyama, S. Shibata, H. Mitsuta, M. Ochi, H. Nakahara, T. Itamoto and T. Asahara, Intraoperative near-infrared spectroscopy for evaluating hepatic venous outflow in living-donor right lobe liver, Transplantation 76(5) (2003), 791-797.

[26] R.S. Prasad, S.J. Smith and H. Wright, Lower abdominal pressure versus external bladder stimulation to aid bladder emptying in multiple sclerosis: a randomized controlled study, Clin. Rehabil. 17(1) (2003), 42-47.

[27] J.C. Puyana, B.R. Soller, S. Zhang and S.O. Heard, Continuous measurement of gut pH with near-infrared spectroscopy during hemorrhagic shock, J. Trauma 46(1) (1999), 9-15.

[28] C. Seif, J. Herzog, C. van der Horst, B. Schrader, J. Volkmann, G. Deuschl, K.P. Juenemann and P.M. Braun, Effect of subthalamic deep brain stimulation on the function of the urinary bladder, Ann. Neurol. 55(1) (2004), 118-120.

[29] N. Shah, A.E. Cerussi, D. Jakubowski, D. Hsiang, J. Butler and B.J. Tromberg, Spatial variations in optical and physiological properties of healthy breast tissue, J. Biomed. Opt. 9(3) (2004), 534-540.

[30] C. Shaw, K. Williams, P.R. Assassa and C. Jackson, Patient satisfaction with urodynamics: a qualitative study, $J . A d v$. Nurs. 32(6) (2000), 1356-1363.

[31] J. Sullivan, P. Lewis, S. Howell, T. Williams, A.M. Shepherd and P. Abrams, Quality control in urodynamics: a review of urodynamic traces from one centre, BJU Int. 91(3) (2003), 201-207.

[32] T. Suto, M. Fukuda, M. Ito, T. Uehara and M. Mikuni, Multichannel near-infrared spectroscopy in depression and schizophrenia: cognitive brain activation study, Biol. Psychiatry 55(5) (2004), 501-511.

[33] F. Steinberg, H.J. Rohrborn, T. Otto, K.M. Scheufler and C. Streffer, NIR reflection measurements of hemoglobin and cytochrome aa3 in healthy tissue and tumors. Correlations to oxygen consumption: preclinical and clinical data, Adv. Exp. Med. Biol. 428 (1997), 69-77.

[34] J.P. Theofrastous, J. Whiteside and M.F. Paraiso, The utility of urodynamic testing, J. Am. Assoc. Gynecol. Laparosc. 10(4) (2003), 548-552.

[35] N. Zelikovsky, J.R. Rodrigue, C.A. Gidycz and M.A. Davis, Cognitive behavioral and behavioral interventions help young children cope during a voiding cystourethrogram, J. Pediatr. Psychol. 25(8) (2000), 535-543. 


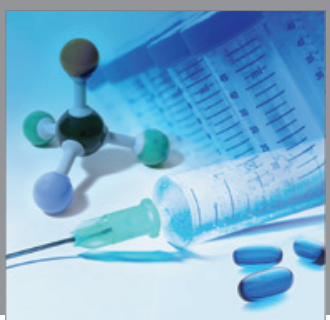

International Journal of

Medicinal Chemistry

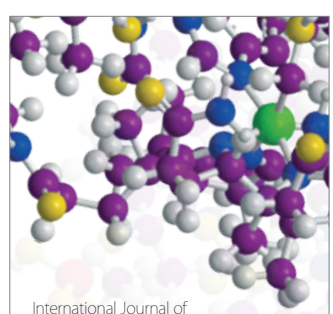

Carbohydrate Chemistry

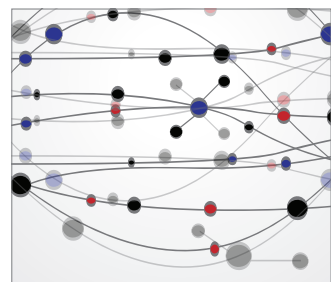

The Scientific World Journal
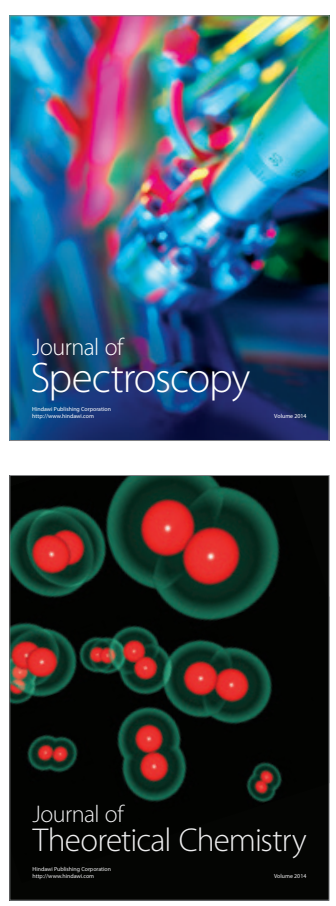
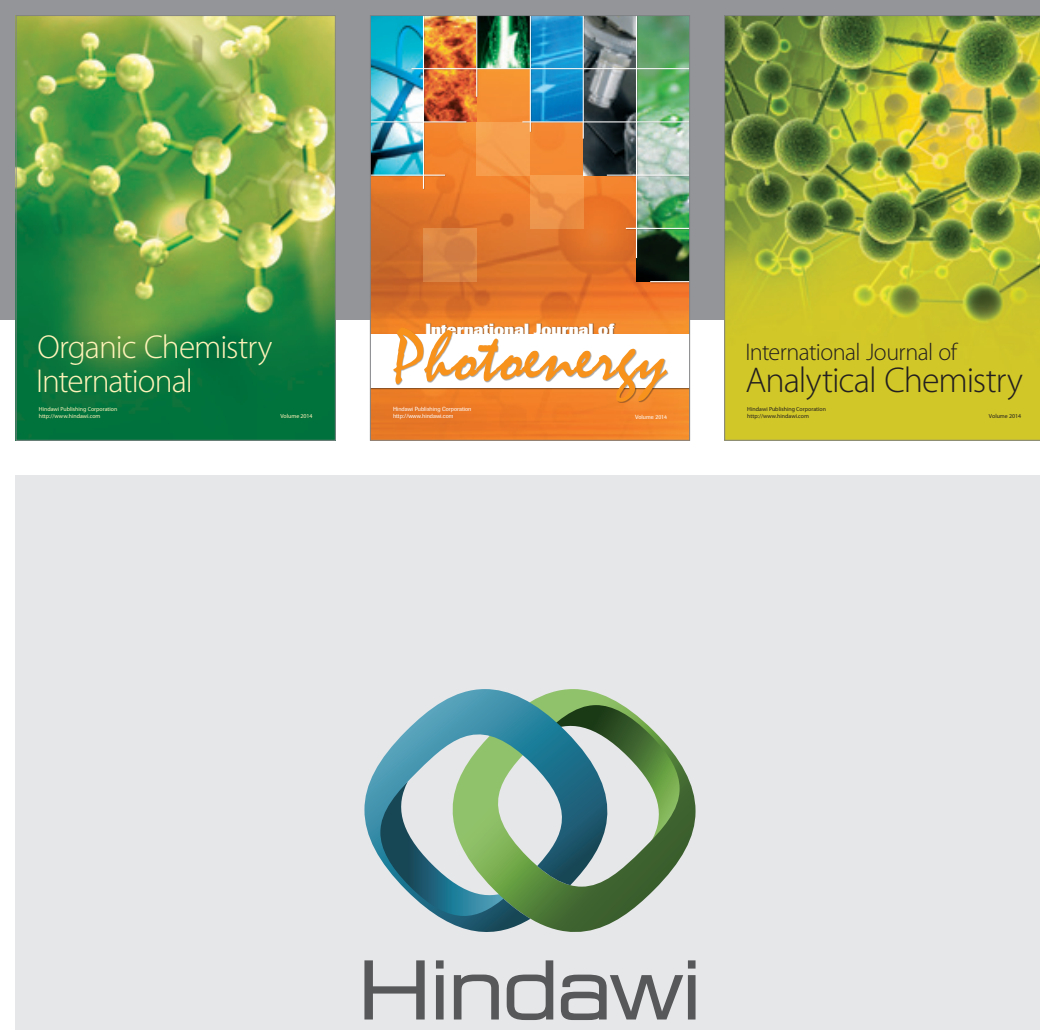

Submit your manuscripts at

http://www.hindawi.com
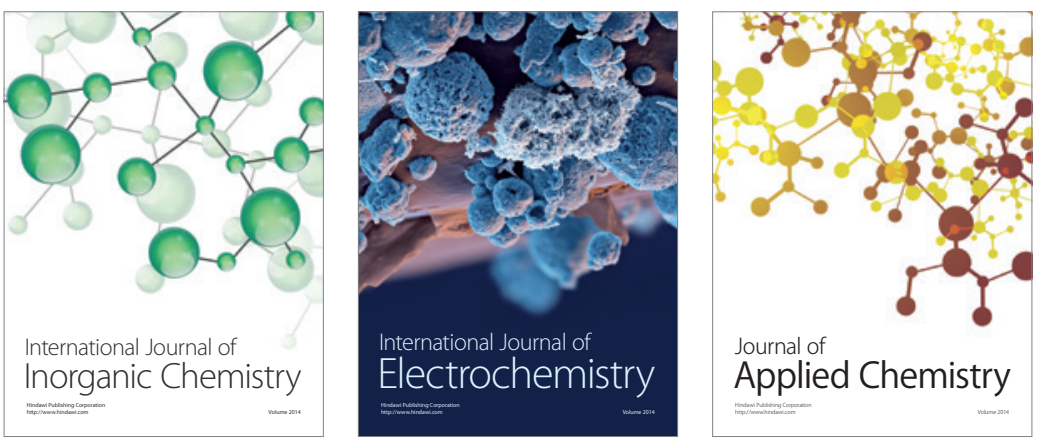

Journal of

Applied Chemistry
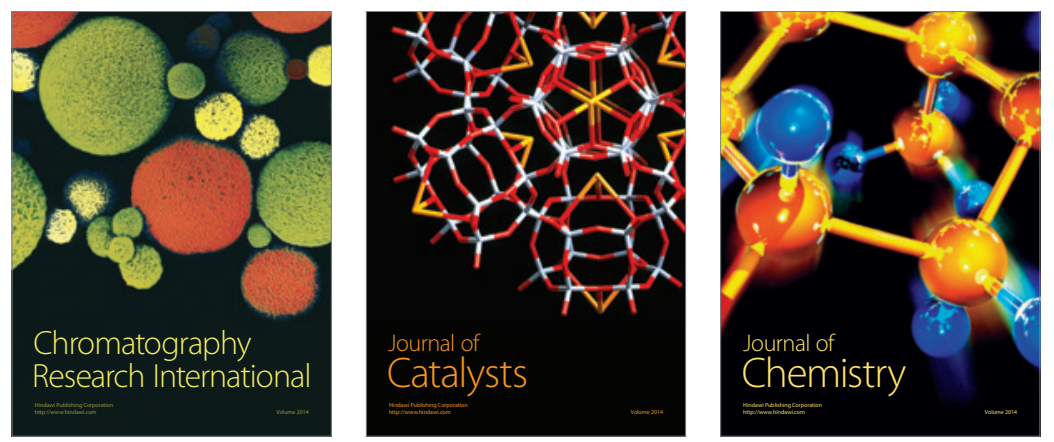
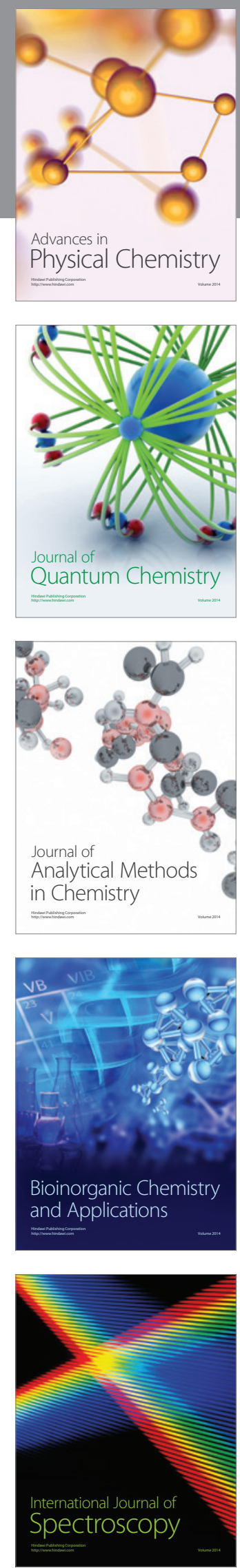